\title{
Generalized measure of quantum synchronization
}

\author{
Noufal Jaseem $\odot,{ }^{1,{ }^{*}}$ Michal Hajdušek, ${ }^{2}$ Parvinder Solanki $\odot,{ }^{1}$ Leong-Chuan Kwek, ${ }^{3,4,5}$ \\ Rosario Fazio, ${ }^{6,7, \dagger}$ and Sai Vinjanampathy $\odot^{1,3}$ \\ ${ }^{1}$ Department of Physics, Indian Institute of Technology-Bombay, Powai, Mumbai 400076, India \\ ${ }^{2}$ Keio University Shonan Fujisawa Campus, 5322 Endo, Fujisawa, Kanagawa 252-0882, Japan \\ ${ }^{3}$ Centre for Quantum Technologies, National University of Singapore, 3 Science Drive 2, 117543 Singapore, Singapore \\ ${ }^{4}$ Institute of Advanced Studies, Nanyang Technological University, Singapore 639673 \\ ${ }^{5}$ National Institute of Education, Nanyang Technological University, Singapore 637616 \\ ${ }^{6}$ ICTP, Strada Costiera 11, 34151 Trieste, Italy \\ ${ }^{7}$ Dipartimento di Fisica, Universit di Napoli Federico II, Complesso di Monte S. Angelo, 80126 - Napoli, Italy
}

(Received 27 June 2020; accepted 26 October 2020; published 30 November 2020)

\begin{abstract}
We present a generalized information-theoretic measure of synchronization in quantum systems. This measure is applicable to dynamics of anharmonic oscillators, few-level atoms, and coupled oscillator networks. Furthermore, the new measure allows us to discuss synchronization of disparate physical systems such as coupled hybrid quantum systems and coupled systems undergoing mutual synchronization that are also driven locally. In many cases of interest, we find a closed-form expression for the proposed measure.
\end{abstract}

DOI: 10.1103/PhysRevResearch.2.043287

\section{INTRODUCTION}

Detecting and measuring synchronization of classical systems has been an important area of research in nonlinear dynamics for decades [1-3]. Regardless of the exact nature of the studied classical system, be it a collection of fireflies, heart cells or firing neurons in the brain, the equations of motion generate trajectories in phase space which enable us to compute an appropriate measure of synchronization.

Quantification of synchronization in quantum systems, on the other hand, does not immediately present such a unified and intuitive approach. Different measures have been introduced to study externally driven and mutually coupled van der Pol oscillators [4-9], driven and coupled spin-1 atoms $[10,11]$, interacting many-body systems [12], spins interacting via coupled optical cavities [13], coupled opto-mechanical systems $[14,15]$, and quantum systems undergoing transient synchronization $[16,17]$. Furthermore, recently such quantum synchronization has been experimentally observed [18,19]. These approaches are in most cases tailored on specific systems. Therefore it would be very interesting to seek a generic quantifier.

In this manuscript, we address this issue by proposing a new measure of quantum synchronization that builds upon insight from quantum information while bringing in several

\footnotetext{
*noufal@iitb.ac.in

On leave
}

Published by the American Physical Society under the terms of the Creative Commons Attribution 4.0 International license. Further distribution of this work must maintain attribution to the author(s) and the published article's title, journal citation, and DOI. desirable new attributes. We exploit the approach that in order to quantify certain quality of a state (e.g., entanglement) one can compute the distance to the nearest state lacking this quality (e.g., set of separable states). This strategy has been extremely fruitful in several contexts such as quantification of entanglement [20-22], discord [23,24], and quantum coherence [25]. We introduce the set of unsynchronized limit-cycle states, the distance to which is then optimized in order to quantify synchronization. They will fulfill a similar role that separable states play in entanglement quantification or that incoherent states play in coherence quantifiction. Note that the unsynchornized limit-cycle states are conceptually different from the notion of a semi-classical limit cycle state used in Refs. $[4,5]$. We show that this approach can be immediately applied in the case of both a single quantum system entrained to an external signal as well as a number of systems undergoing mutual synchronization. Furthermore, we prove that our synchronization measure works for finite and infinite-dimensional systems. Finally, we demonstrate that by construction the limit-cycle state allows for great flexibility and can even be applied to study synchronization of disparate quantum systems, as depicted in Fig. 1 .

In the next section, we define our new measure of synchronization. In Sec. III, we review two canonical examples of systems studied in quantum synchronization. In Secs. IV and $\mathrm{V}$, we apply our measure to unipartite and bipartite systems, respectively, along with relevant examples. Section VI provides concluding discussion.

\section{RELATIVE ENTROPY OF SYNCHRONIZATION}

In analogy to classical dynamical systems, synchronization in quantum systems begins by establishing the existence of a limit cycle. In classical mechanics, establishing a limit cycle 
(a)

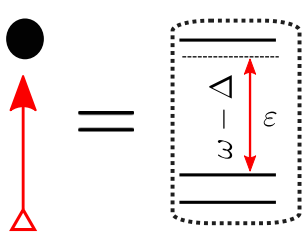

(c)

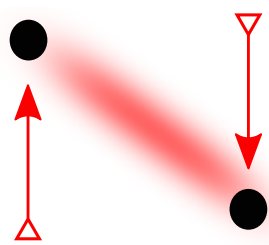

(b)

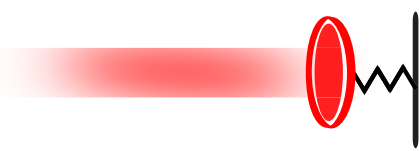

(d)

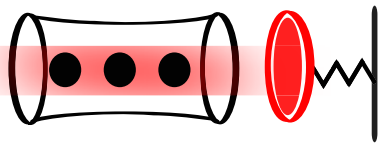

FIG. 1. A glimpse of the variety of systems that can be studied using the distance-based measure introduced in this paper. (a) Driven three-level system. (b) Driven quantum vdP. (c) Two driven threelevel systems which are coupled to each other. (d) Quantum vdP coupled to mutually interacting three-level systems.

implies that the relevant free coordinates are identified, which in the case of a van der Pol oscillator for example is its phase [2]. Once the limit cycle is established, the dynamical systems may be (a) entrained to an external frequency standard or (b) coupled with each other in order to observe mutual synchronization.

The common physical insight that goes into defining the different measures of synchronization can be summarized as (a) identifying the limit-cycle behavior in the quantum system and (b) quantifying deviations from such a state. Motivated by the current discussion, a natural choice of quantifying this deviation is by considering the distance $\mathfrak{D}\left(\rho, \rho_{\text {lim }}\right)$ between the steady state $\rho$ of the evolution and its limit-cycle state $\rho_{\text {lim }}$. The distance $\mathfrak{D}$ in fact does not need to be a strict measure but can also be distancelike as we will demonstrate in this section.

A question that remains is what is the appropriate limitcycle state $\rho_{\text {lim }}$ that can be used to quantify synchronization. In general, it is not the steady state of the evolution $\dot{\rho}=\mathcal{L}[\rho]$ where the external drive $\varepsilon$ and the mutual coupling $g$ have been switched off. This is because the external drive and mutual coupling have two disparate effects. First, they produce entrainment/synchronization that is of interest to us. Besides this, they also may change the population of the system which does not affect the synchronization properties of the system. Proper measure of synchronization should quantify only the first effect and be insensitive to the latter. We demonstrate these two effects schematically on an externally driven van der Pol oscillator in Fig. 2.

In order to quantify the synchronization of a steady state $\rho$ we must minimize the distance $\mathfrak{D}$ over all possible limitcycle states, leading to the definition of our new measure of synchronization,

$$
\Omega(\rho) \equiv \min _{\sigma \in \Sigma} \mathfrak{D}(\rho, \sigma)
$$

where $\Sigma$ is the set of all possible limit-cycle states.
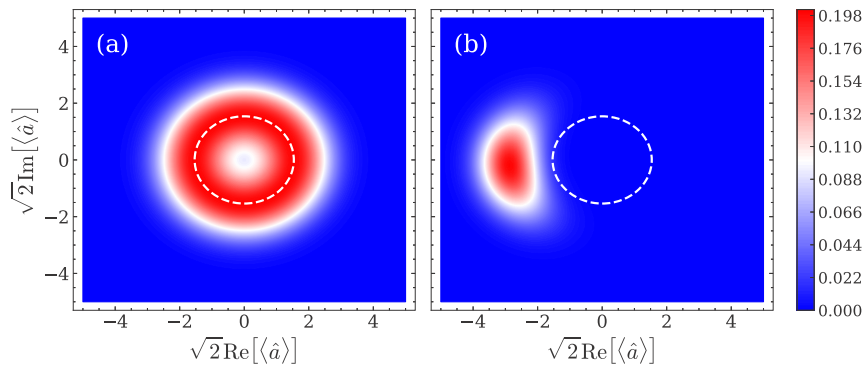

FIG. 2. (a) Wigner function of the steady state of an undriven van der Pol oscillator of Eq. (4). Parameters used are $\Delta / \Gamma_{g}=0.1$, $\Gamma_{d} / \Gamma_{g}=0.5$, and $\varepsilon / \Gamma_{g}=0$. (b) The external drive entrains the oscillator, as seen by the localization of the Wigner function, as well as changes its population distribution. The white dashed circle in (a) and (b) depicts the average population of an undriven oscillator. Parameters are the same except for $\varepsilon / \Gamma_{g}=3$. Note that the driving strength has been set this high in order to demonstrate the effect of population change clearly. Synchronization is usually limited to perturbative driving where the effect of population change is much smaller.

We consider two important examples of distance functions $\mathfrak{D}$. The first one is the relative entropy of synchronization,

$$
\Omega_{\mathrm{R}}(\rho) \equiv \min _{\sigma \in \Sigma} S(\rho \| \sigma),
$$

where $S(\rho \| \sigma)=\operatorname{Tr}[\rho \ln \rho-\rho \ln \sigma]$. This measure is suitable when $\sigma$ is full rank which is true in the cases that we consider. In order to handle limit cycles which are not full rank we propose the trace distance to quantify synchronization,

$$
\Omega_{\mathrm{D}}(\rho) \equiv \min _{\sigma \in \Sigma}\|\rho-\sigma\|_{1},
$$

where $\|O\|_{1}=\operatorname{Tr}\left[\sqrt{O^{\dagger} O}\right]$.

The set of limit-cycle states $\Sigma$ depends on two things. First, it depends on the particular dynamics under investigation. The form of the limit-cycle state $\rho_{\text {lim }}$ will be different for a single system driven externally and for two coupled systems undergoing mutual synchronization. Second, we might be interested in more complex scenarios where for instance two coupled systems are also being locally driven. In such cases the limitcycle states require a suitable form that reflects not only the dynamics being studied but also that distinguishes between synchronization due to mutual coupling and entrainment due to the local drives.

In order for our proposed measure in Eq. (1) to be a physically reasonable quantity the form of the limit-cycle states must be such that the already existing measures of synchronization vanish for these states. This will be the guiding principle in defining three classes of limit-cycle states which we study. Two of these classes are inspired by existing results while the third class is newly defined to specifically demonstrate the flexibility of our proposed measure.

Diagonal limit-cycle states. It follows from the definition of a free observable phase that a self-sustaining system which is not driven and not coupled to other systems will be diagonal in the energy eigenbasis. Diagonal limit-cycle states are useful when studying entrainment of a single quantum system to an external drive $[4,10]$ as well as mutual synchronization of coupled systems $[11,15]$. They are physically motivated 
by the fact that existing measures all vanish for diagonal states. Furthermore, their simple form allows us to find a closed form for the minimization in Eq. (1). Note that whether diagonal states are valid limit-cycle states has to be motivated from the physics of the problem. As mentioned before, the existence of a stable limit cycle is a necessary condition for synchronization, implying the existence of inherent or acquired nonlinearity. For instance, consider a single-mode bosonic field undergoing damping due to a thermal bath at temperature $T$. The steady state is given by a thermal state which is diagonal in the energy eigenbasis but is not selfoscillating since it lacks the requisite nonlinearity. The Wigner function of such a thermal state is a Gaussian centered at the origin and does not possess the required limit cycle as seen in Fig. 2(a).

Marginal limit-cycle states. Mutual information is well defined both in the classical and quantum world, making it an appropriate measure of quantum systems which can exhibit synchronization both in the classical and quantum regime. Motivated by this, authors in Ref. [13] introduced this class of limit-cycle states to study coupled van der Pol oscillators. Marginal limit-cycle states do not necessarily need to be diagonal making them more general when compared with diagonal limit-cycle states. They were motivated physically showing that the mutual information measure vanishes when measure of complete synchronization in Ref. [13] does as well.

Partially-coherent limit-cycle states. This previously unstudied class of limit-cycle states is the third example considered in this manuscript. These states offer a large degree of flexibility to the form of the limit-cycle state over which one minimizes the distance measures and is particularly suited to situations where there are a number of sources that may entrain or synchronize a system and our aim is to isolate a subset of these sources. Therefore they are even more general than marginal limit-cycle states since they offer direct control over the coherences present in the state. An example of such scenario is given in Eq. (9) by two coupled spin-1 atoms which are also locally driven.

In the following sections, we demonstrate how these three classes of limit-cycle states are used in various scenarios and derive some useful properties of the new measure of synchronization. Before we proceed, we would like to reiterate the importance of knowing the underlying physical model prior to applying our synchronization measure in Eq. (1). Consider the case of a single-mode bosonic field undergoing thermalization from above, and add a coherent drive of appropriate frequency. Our synchronization measure will register a finite value even though the phenomenon that this system displays is resonance rather than synchronization. This holds irrespective of the measure of synchronization and whether it is quantum or classical [2]. Therefore it is crucial to first identify a suitable dynamical system that is capable of synchronization and then apply the measure to discover how much the system is synchronized.

\section{EXAMPLES OF SYNCHRONIZING SYSTEMS}

The quantum systems undergoing synchronization can be split into two groups, continuous variable systems and finite-dimensional systems. We focus on one canonical representatives from each group. In this section, we outline the van der Pol oscillator, a continuous variable model, while the finite-dimensional systems are represented by a spin-1 atom. Both of these systems can be used to study entrainment of a single oscillator to an external drive as well as mutual synchronization of a number of coupled oscillators.

Continuous variable systems. Infinite-dimensional systems are a natural candidate to study synchronization since they follow in close analogy from their classical counterparts. Following this intuition, the authors in Refs. [4,5] studied a driven harmonic oscillator mode undergoing Lindblad dynamics due to two baths,

$$
\dot{\rho}=-i[\hat{H}, \rho]+\Gamma_{g} \mathcal{D}\left[\hat{a}^{\dagger}\right] \rho+\Gamma_{d} \mathcal{D}\left[\hat{a}^{2}\right] \rho .
$$

Here, $\hat{H}=-\Delta \hat{a}^{\dagger} \hat{a}+i \varepsilon\left(\hat{a}-\hat{a}^{\dagger}\right)$ with $\Delta$ being the detuning of the drive's frequency $\omega_{d}$ from the natural frequency of the oscillator $\omega_{0}$, and $\mathcal{D}[\hat{O}] \rho=\left(2 \hat{O} \rho \hat{O}^{\dagger}-\hat{O}^{\dagger} \hat{O} \rho-\rho \hat{O}^{\dagger} \hat{O}\right) / 2$. Note that just like classical dynamical systems require a source and sink of energy to compete in a nonlinear manner in order to form a limit cycle, Eq. (4) has two dissipators, one that removes two excitations incoherently from the system and another that adds one excitation incoherently to the system. This ensures that the amplitude of oscillations is stable and the quantum van der Pol oscillator possesses a limit cycle.

Two van der Pol oscillators can be coupled in a number of possible ways. Authors in Ref. [5] considered a coherent coupling by adding an interaction Hamiltonian,

$$
\hat{H}_{\text {int }}=g\left(\hat{a}_{1}^{\dagger} \hat{a}_{2}+\hat{a}_{1} \hat{a}_{2}^{\dagger}\right) \text {. }
$$

Another possibility is to couple the two oscillators dissipatively by adding another Lindblad operator as in Refs. $[8,26]$,

$$
\dot{\rho}=\mathcal{L}_{1}[\rho]+\mathcal{L}_{2}[\rho]+g \mathcal{D}\left[\hat{a}_{1}-\hat{a}_{2}\right] \rho,
$$

where $\mathcal{L}_{i}[\rho]$ is the Lindblad evolution given by Eq. (4) with $\varepsilon=0$.

Finite-level systems. Investigation of synchronization in the quantum regime motivated the study of small quantum systems with no classical analogs. Authors in Ref. [10] explored an externally driven spin-1 atom. In the frame rotating with the external signal,

$$
\dot{\rho}=-i[\hat{H}, \rho]+\frac{\gamma_{g}}{2} \mathcal{D}\left[\hat{S}_{+} \hat{S}_{z}\right] \rho+\frac{\gamma_{d}}{2} \mathcal{D}\left[\hat{S}_{-} \hat{S}_{z}\right] \rho,
$$

where $\hat{H}=\Delta \hat{S}_{z}+\varepsilon \hat{S}_{y}$ and $\hat{S}_{ \pm}$are the raising/lowering operators. The limit cycle is established by the nonlinear nature of the dissipator operators stabilizing the middle energy level. This concentrates the whole atomic population into the middle level meaning the limit-cycle state is pure unlike in the case of a van der Pol oscillator. General framework to study entrainment of spin-1 atoms to an external signal was presented in Ref. [27].

Mutual synchronization of two such spin-1 systems was considered in Ref. [11] by introducing a coherent interaction term,

$$
\hat{H}_{\mathrm{int}}=i g\left(\hat{S}_{-}^{A} \hat{S}_{+}^{B}-\hat{S}_{+}^{A} \hat{S}_{-}^{B}\right),
$$

in the context of entanglement production in the steady state.

In order to demonstrate the flexibility of our proposed measure of synchronization, we also consider a system of two 
coherently coupled spin-1 atoms where each subsystem is also driven locally,

$$
\dot{\rho}=-i[\hat{H}, \rho]+\sum_{\alpha=A, B}\left\{\frac{\gamma_{g}^{\alpha}}{2} \mathcal{D}\left[\hat{S}_{+}^{\alpha} \hat{S}_{z}^{\alpha}\right]+\frac{\gamma_{d}^{\alpha}}{2} \mathcal{D}\left[\hat{S}_{-}^{\alpha} \hat{S}_{z}^{\alpha}\right]\right\} \rho,
$$

where the coherent part of the evolution is given by

$$
\begin{aligned}
\hat{H}= & \delta \hat{S}_{z}^{A}+(\delta+\Delta) \hat{S}_{z}^{B}+\epsilon \sum_{\alpha=A, B}\left(\hat{S}_{z}^{\alpha} \hat{S}_{+}^{\alpha}+\hat{S}_{-}^{\alpha} \hat{S}_{z}^{\alpha}\right) \\
& +i g\left(\hat{S}_{+}^{A} \hat{S}_{-}^{B}-\hat{S}_{-}^{A} \hat{S}_{+}^{B}\right),
\end{aligned}
$$

with $\delta$ being the detuning of the local drives from the natural frequencies of the spin- 1 atoms and $\Delta$ the detuning between the atoms.

\section{UNIPARTITE SYSTEMS}

Let us consider the scenario where a single quantum system is entrained to an external drive [4,5]. In the absence of the external drive the steady state of the evolution is diagonal in the energy eigenbasis $\left\{\left|E_{i}\right\rangle\right\}$. Introducing the external drive has two consequences. First, it produces a change in the populations given by the diagonal elements of the steady-state density matrix. Second, it populates the off-diagonal elements of the steady-state density matrix $\rho$ and may produce entrainment to the external drive.

Consider a diagonal limit-cycle state,

$$
\rho_{\lim }=\sum_{j} q_{j}\left|E_{j}\right\rangle\left\langle E_{j}\right|
$$

The relative entropy $S\left(\rho \| \rho_{\text {lim }}\right)$ can be expanded as

$$
S\left(\rho \| \rho_{\lim }\right)=S_{\mathrm{coh}}(\rho)+D_{K L}[p \| q],
$$

where $S_{\mathrm{coh}}(\rho)=S\left(\rho_{\mathrm{diag}}\right)-S(\rho)$ is the relative entropy of coherence [25], $S(\rho)=-\operatorname{Tr}[\rho \ln \rho]$ is the von Neumann entropy, and $\rho_{\text {diag }}$ is obtained from $\rho$ by setting its off-diagonal terms to zero. The second term in Eq. (12) given by the Kullback-Leibler divergence of populations of the steady state $\rho$ and the limit-cycle state $\rho_{\lim }$ describes the change in the population of the driven system mentioned in Sec. II and pictured in Fig. 2.

The minimization of Eq. (12) over all diagonal limit-cycle states can be done simply by setting $\rho_{\text {lim }}=\rho_{\text {diag }}$,

$$
\Omega_{\mathrm{R}}(\rho)=S_{\operatorname{coh}}(\rho) .
$$

Equation (13) provides a closed-form expression for the minimization problem in Eq. (2) and shows that relative entropy of synchronization and relative entropy of coherence are equivalent when considering a single quantum system driven externally. In Fig. 3(a), we plot $\Omega_{\mathrm{R}}(\rho)$ of a harmonically driven van der Pol oscillator by numerically solving Eq. (4) for its steady state using QuTip [28,29]. We observe the usual Arnold tongue as expected from a well-behaved measure of synchronization. In Fig. 3(b), we compare this against $C_{1}(\rho)=|\langle\hat{a}\rangle| / \sqrt{\left\langle\hat{a}^{\dagger} \hat{a}\right\rangle}$, which is a synchronization measure used in Ref. [15].

Using a similar strategy to perform the minimization we can find an upper bound on the trace distance measure of
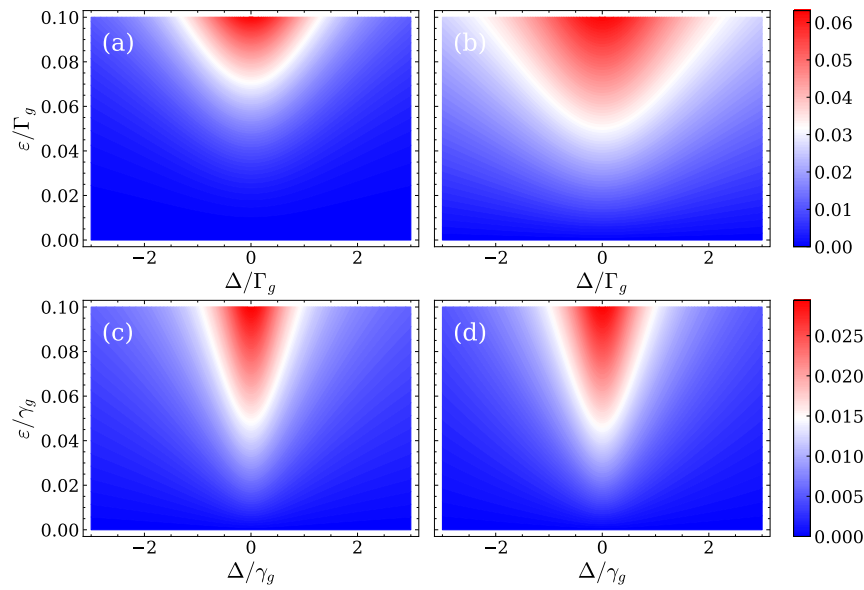

FIG. 3. Relative entropy of synchronization $\Omega_{R}(\rho)$ of an externally driven van der Pol oscillator in (a) displays qualitatively same behavior as $C_{1}(\rho)$ measure of synchronization of Ref. [15] shown in (b). The damping rates are $\Gamma_{d} / \Gamma_{g}=10$. (c) Trace distance measure of synchronization $\Omega_{\mathrm{D}}(\rho)$ for an externally driven spin-1 atom. (d) Phase-locking measure $S_{\text {phase }}(\rho)$ of Ref. [10]. The damping rates for the spin- 1 atom are $\gamma_{d} / \gamma_{g}=10$.

synchronization $\Omega_{\mathrm{D}}$. The trace distance satisfies the following inequality:

$$
\left\|\rho-\rho_{\lim }\right\|_{1} \leqslant \sum_{j}\left|p_{j}-q_{j}\right|+\sum_{j \neq k}\left|\rho_{j k}\right| .
$$

Setting $\rho_{\text {lim }}=\rho_{\text {diag }}$ in Eq. (14), we obtain an upper bound on the trace distance measure of synchronization,

$$
\Omega_{\mathrm{D}}(\rho) \leqslant C_{l_{1}}(\rho),
$$

where $C_{l_{1}}(\rho)=\sum_{j \neq k}\left|\rho_{j k}\right|$ is the $l_{1}$-norm of coherence introduced in [25]. Figure 3(c) shows the Arnold tongue of $\Omega_{\mathrm{D}}(\rho)$ for a driven spin-1 atom model of Eq. (7). For reference we display the phase measure of synchronization $S_{\text {phase }}(\rho)$ defined in Eq. (8) of Ref. [10].

The connection between synchronization and coherence has been noted previously in literature, for example, in Refs. [27,30]. In fact, for some existing measures one can quickly see that they vanish when the steady state $\rho$ is diagonal in the energy eigenbasis. The result of Eq. (13) is the first time when it was shown that a measure of synchronization reduces exactly to one of the well-established measures of coherence. On the other hand we will show in the next section that this is not the case anymore for general bipartite synchronization, demonstrating that synchronization is generally not equivalent to coherence.

\section{BIPARTITE SYSTEMS}

In this section, we apply our measure to bipartite systems and study their mutual synchronization. The minimization in Eq. (2) for general limit-cycle states $\sigma=\sum_{i} q_{i} \sigma_{i}^{A} \otimes \sigma_{i}^{B}$, where $\sigma_{i}^{A / B}$ are the limit-cycle states for the individual 
subsystems, proceeds as follows:

$$
\begin{aligned}
\Omega_{\mathrm{R}}(\rho)= & \min _{\sigma \in \Sigma} S(\rho \| \sigma) \\
= & -S(\rho)-\max _{q_{i}, \sigma_{i}^{A}, \sigma_{i}^{B}} \operatorname{Tr}\left[\rho \ln \left(\sum_{i} q_{i} \sigma_{i}^{A} \otimes \sigma_{i}^{B}\right)\right] \\
\leqslant & -S(\rho)-\max _{q_{i}, \sigma_{i}^{A}} \sum_{i} q_{i} \operatorname{Tr}\left[\rho_{A} \ln \sigma_{i}^{A}\right] \\
& -\max _{q_{i}, \sigma_{i}^{B}} \sum_{i} q_{i} \operatorname{Tr}\left[\rho_{B} \ln \sigma_{i}^{B}\right] \\
\leqslant & -S(\rho)-\omega_{A}-\omega_{B}
\end{aligned}
$$

where we defined $\omega_{\alpha}=\max _{q_{i}, \sigma_{i}^{\alpha}} \sum_{i} q_{i} \operatorname{Tr}\left[\rho_{\alpha} \ln \sigma_{i}^{\alpha}\right]$. In the above, we have used the concavity of the expression, $\operatorname{Tr}\left[\rho \ln \left(\sum_{i} q_{i} \sigma_{i}\right)\right]$ as $\operatorname{Tr}\left[\rho \ln \left(\sum_{i} q_{i} \sigma_{i}\right)\right] \geqslant \sum_{i} q_{i} \operatorname{Tr}\left[\rho \ln \sigma_{i}\right]$ for $0<q_{i}<1$ and $\sum_{i} q_{i}=1$. This inequality saturates when $q_{1}=1$ and the rest of $q_{i}$ 's are zero. Though analytically evaluating $\omega_{\alpha}$ is difficult for general limit-cycle states, we show in the rest of this section that for many classes of limit-cycle states it is possible to find closed form expressions for the minimization in Eq. (2).

\section{A. Diagonal limit-cycle states}

Owing to classical correlations, the set of diagonal limit cycle states denoted as $\delta$ is more general than just the tensor product of diagonal states. The relative entropy of synchronization for the set of diagonal limit-cycle states can be evaluated to be

$$
\begin{aligned}
\Omega_{\mathrm{R}}(\rho) & =\min _{\delta} S(\rho \| \delta) \\
& =\min _{\delta}\left[S_{\mathrm{coh}}(\rho)+S\left(\rho_{\text {diag }} \| \delta\right)\right] \\
& =S_{\mathrm{coh}}(\rho) .
\end{aligned}
$$

The minimization of the relative entropy of synchronization over $\delta$ yields the relative entropy of coherence, similar to our discussion in the unipartite case. This result is obtained when the set of diagonal limit-cycle states is not restricted to a simple tensor product. Convex mixtures of diagonal limitcycle states are themselves diagonal-limit cycle states and hence they do not produce any synchronization. The physical motivation for including these convex mixtures of limit-cycle states is that if two quantum systems are coupled via operators that are diagonal with respect to the eigenbasis of the undriven steady states (either in the Hamiltonian or Lindbladians), while these couplings can give rise to correlations, they cannot generate synchronization. We account for the buildup of such classical correlations in this scenario by optimizing over the full set of diagonal states.

To further illustrate this point, consider a scenario where the limit-cycle states are composed of uncorrelated diagonal states, namely $\delta=\delta^{A} \otimes \delta^{B}$. Such a physical scenario arises when two hitherto noninteracting quantum systems are coupled by a synchronizing coupling either in the Hamiltonian [5] or Lindbladians [8]. Since they are noninteracting without the synchronizing coupling, their limit-cycle states will be uncorrelated. Minimization over such local diagonal limit-cycle states yields

$$
\min _{\delta^{A}, \delta^{B}} S\left(\rho \| \delta^{A} \otimes \delta^{B}\right)=S_{\mathrm{coh}}(\rho)+I_{c}(\rho),
$$

where $I_{c}(\rho)=S\left(\rho_{\text {diag }}^{A}\right)+S\left(\rho_{\text {diag }}^{B}\right)-S\left(\rho_{\text {diag }}\right)$ is the classical mutual information. Since $I_{c}(\rho) \geqslant 0$, we see that the additional classical correlations developed by the uncoupled systems are properly accounted for in the synchronization measure. This same term $I_{c}(\rho)$ is removed when the limit cycles are classically correlated in Eq. (17)

As an example, we considered two mutually coupled three-levels systems that were studied in Ref. [11]. The corresponding relative entropy of synchronization is depicted in the Fig. 4(a), which shows agreement with known results.

\section{B. Synchronization of dissimilar systems}

Until now, the study of mutual synchronization has been restricted to coupling identical systems $[5,8,13]$. In contrast to this, quantum technology platforms such as quantum optics, cavity and circuit quantum electrodynamics and many-body physics routinely deal with coupling dissimilar systems. As a prototypical example, let us consider the Tavis-Cummings model whose Hamiltonian within rotating wave approximation is given by

$$
\hat{H}=\Omega \hat{J}_{z}+\omega\left(\hat{a}^{\dagger} \hat{a}+\frac{1}{2}\right)+\epsilon\left(\hat{a} \hat{J}_{+}+\hat{a}^{\dagger} \hat{J}_{-}\right) .
$$

Here, $\hat{J}_{i}=\sum_{\alpha} \sigma_{\alpha}^{(i)} / 2$ are the collective spins of $N$ atoms and $\hat{a}$ is a bosonic annihilation operator. In the large $N$ limit, this Hamiltonian could be transformed into a coupled bosonic Hamiltonian by employing the Holstein-Primakoff transformation. If we discuss the synchronization of such systems, once an underlying limit cycle has been established, we could employ the measures reviewed earlier to propose a measure of synchronization based on the relative phase. On the other hand, if we wish to discuss the $N \approx 1$ limit of the TavisCummings model, then all previous measures fail. In contrast to this, our new measure can handle the synchronization of such a dissimilar systems without any further modifications.

As an example of the synchronization of dissimilar quantum systems, consider a van der Pol oscillator coupled to an equally spaced three-level system. The Hamiltonian for the combined system in the rotated frame is given by

$$
\hat{H}_{R}=\hat{H}_{0}+\epsilon\left(\hat{S}_{+} \hat{a}+\hat{a}^{\dagger} \hat{S}_{-}\right),
$$

where the free Hamiltonian is $\hat{H}_{0}=\left(\omega_{a}+\Delta\right) \hat{S}_{z}+\omega_{a} \hat{a}^{\dagger} \hat{a}, \omega_{a}$ is the natural frequency of the van der Pol oscillator, $\Delta$ is the detuning between the spin-1 system and the oscillator, and $g$ is the coupling strength. The Lindblad master equation for the combined state $\rho$ is given by

$$
\begin{aligned}
\dot{\rho}= & -i[\hat{H}, \rho]+\gamma_{d} \mathcal{D}\left[\hat{S}_{-} \hat{S}_{z}\right] \rho+\gamma_{g} \mathcal{D}\left[\hat{S}_{+} \hat{S}_{z}\right] \rho \\
& +\Gamma_{d} \mathcal{D}\left[\hat{a}^{2}\right] \rho+\Gamma_{g} \mathcal{D}\left[\hat{a}^{\dagger}\right] \rho .
\end{aligned}
$$

where $\Gamma_{g}\left(\gamma_{g}\right)$ and $\Gamma_{d}\left(\gamma_{d}\right)$ are the pumping and damping constants for the van der Pol and spin-1 atom, respectively. Such a system represents a van der Pol oscillator coupled to a threelevel system, both of which have been individually discussed in the literature before but never coupled.

The minimization of the relative entropy of synchronization in this case is performed over all possible diagonal limit-cycle states, and hence this example reduces to the 

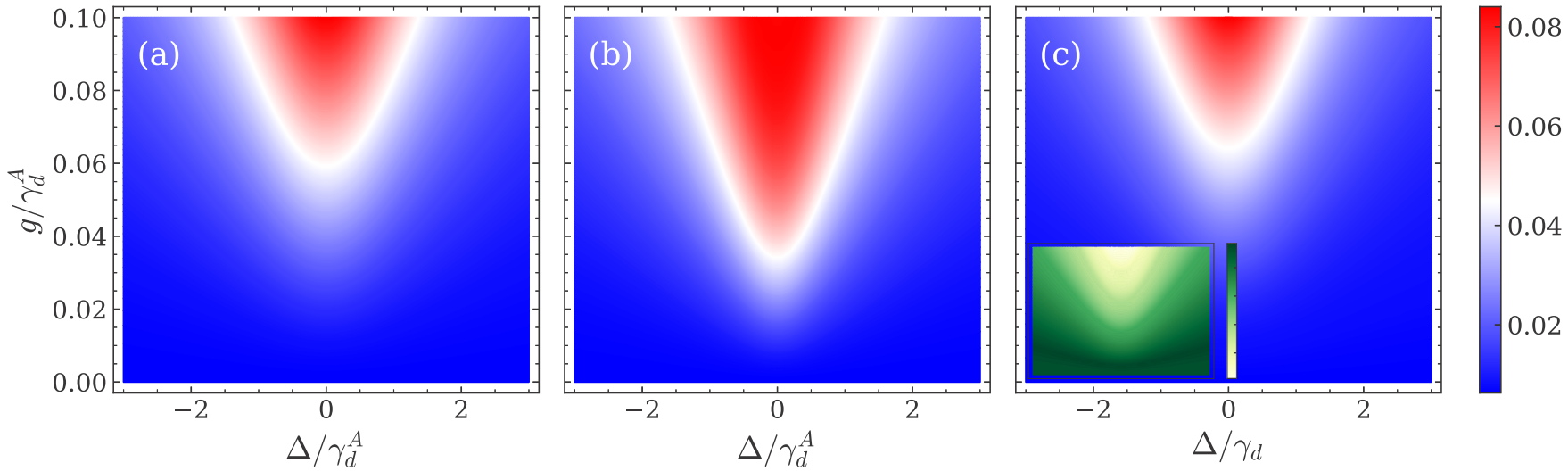

FIG. 4. Relative entropy of synchronization $\Omega_{\mathrm{R}}(\rho)$ for two mutually coupled systems. (a) Two spin-1 atoms coupled coherently as in Eq. (8). The dissipators are $\gamma_{g}^{A} / \gamma_{d}^{A}=\gamma_{d}^{B} / \gamma_{d}^{A}=100$ and $\gamma_{g}^{B}=\gamma_{d}^{A}$. (b) Spin-1 atom coupled to a van der Pol oscillator as in Eq. (21). The parameters are $\Gamma_{d} / \gamma_{d}=0.1, \gamma_{g} / \gamma_{d}=100$, and $\Gamma_{g}=\gamma_{d}$ (c). Two coupled spin-1 atoms that are also driven locally as in Eq. (9). The dissipators are the same as in (a), the local drives are resonant with the atoms, $\delta / \gamma_{d}^{A}=0$, and their strength is $\varepsilon / \gamma_{d}^{A}=0.01$. The effect of the local drives is to decrease mutual synchronization between the atoms. Qualitative difference between $\Omega_{R}$ for partially-coherent limit cycles and the mutual information is shown in the inset.

discussion of Sec. V A. We consequently obtain $\Omega_{R}=S_{\text {coh }}$, and this is plotted as a function of detuning $\Delta$ and the coupling strength $\epsilon$ in Fig. 4(b).

\section{Marginal state limit cycles}

We now consider limit-cycle states which are not necessarily diagonal in the energy eigenbasis. In order to obtain a closed-form expression for $\Omega_{\mathrm{R}}(\rho)$, we consider an example where the two limit-cycle states are uncorrelated. The full limit-cycle state is then given by

$$
\sigma=\sigma^{A} \otimes \sigma^{B}, \quad \text { where } \quad \sigma^{\alpha}=\sum_{i j} q_{i j}^{\alpha}\left|E_{i}^{\alpha}\right\rangle\left\langle E_{j}^{\alpha}\right| .
$$

Substituting this state into (16),

$$
\Omega_{\mathrm{R}}(\rho)=\min _{\sigma}\left\{-S(\rho)-\sum_{\alpha} \operatorname{Tr}\left[\rho \ln \sigma^{\alpha}\right]\right\} .
$$

Adding and subtracting the von Neumann entropies of the marginal states of $\rho$,

$$
\Omega_{\mathrm{R}}(\rho)=\min _{\sigma^{A}, \sigma^{B}}\left\{-S(\rho)+\sum_{\alpha}\left[S\left(\rho^{\alpha}\right)+S\left(\rho^{\alpha} \| \sigma^{\alpha}\right)\right]\right\} .
$$

As $S\left(\rho^{\alpha} \| \sigma^{\alpha}\right) \geqslant 0$, the minimum value is obtained when $\sigma^{\alpha}=$ $\rho^{\alpha}$, yielding

$$
\Omega_{\mathrm{R}}(\rho)=-S(\rho)+S\left(\rho^{A}\right)+S\left(\rho^{B}\right) .
$$

This shows that for the set of limit-cycle states given by uncorrelated nondiagonal states the closest states to the steady state $\rho$ are given by its marginals. In turn, this means that the relative entropy of synchronization $\Omega_{R}(\rho)$ is given by the mutual information between the two systems.

This result is consistent with [13], where the authors proposed mutual information as an order parameter for two mutually coupled van der Pol oscillators. However, note that the mutual information was postulated in Ref. [13] to be a suitable measure by showing that it shared qualitative features with a physically motivated measure of complete synchronization. We formalize this in Eq. (25) by showing that mutual information is the only possible measure when minimizing over the set of limit-cycle states in Eq. (22).

\section{Partially coherent limit-cycle states}

We now consider a new category of limit-cycle states that have not been considered in literature before, namely limit-cycle states that are partially coherent. This interpolates between diagonal limit-cycle states, where there are no offdiagonal terms present and marginal limit-cycle states, where all off-diagonal terms in the marginal density matrix could be nonzero. This intermediate family of limit-cycle states allows us to discuss, for example, locally driven systems which are then coupled to each other. Consider two spin-1 atoms which are locally driven across the $\left|E_{2}\right\rangle\left\langle E_{3}\right|$ transition. A generic representation of such a qutrit is written as $\sigma=$ $\sum_{i} q_{i}\left|E_{i}\right\rangle\left\langle E_{i}\left|+q_{23}\right| E_{2}\right\rangle\left\langle E_{3}\left|+q_{32}\right| E_{3}\right\rangle\left\langle E_{2}\right|$ and constitutes our intermediate limit-cycle state. If we now couple these two qutrits, our new measure can reveal any subsequent mutual synchronization of systems without including any possible entrainment due to these local drives.

Consider a diagonal marginal state, $\lambda=\sum_{i} \lambda_{i}|i\rangle\langle i|$. By applying a general unitary which acts on the subspace $\left\{\left|E_{2}\right\rangle,\left|E_{3}\right\rangle\right\}$, a general partially coherent limit-cycle state can be generated as $\sigma^{\alpha}=U \lambda U^{\dagger}=\sum_{k=1}^{3} q_{k} U|k\rangle\langle k| U^{\dagger}$, where $U=1 \oplus u$ and the unitary $u\left(\theta_{1}, \theta_{2}, \theta_{3}\right)$ which acts on the subspace $\left\{\left|E_{2}\right\rangle,\left|E_{3}\right\rangle\right\}$ is given by

$$
\begin{aligned}
u\left(\theta_{1}, \theta_{2}, \theta_{3}\right) & =e^{-i \theta_{1} \hat{\sigma}_{z}} e^{-i \theta_{2} \hat{\sigma}_{x}} e^{-i \theta_{3} \hat{\sigma}_{z}} \\
& =\left(\begin{array}{cc}
e^{-i\left(\theta_{1}+\theta_{3}\right)} \cos \theta_{2} & -i e^{-i\left(\theta_{1}-\theta_{3}\right)} \sin \theta_{2} \\
-i e^{i\left(\theta_{1}-\theta_{3}\right)} \sin \theta_{2} & e^{i\left(\theta_{1}+\theta_{3}\right)} \cos \theta_{2}
\end{array}\right) .
\end{aligned}
$$

Again we can calculate,

$$
\begin{aligned}
\omega_{\alpha}= & \max _{p_{k}, u} \sum_{k} \ln q_{k}^{\alpha}\left\langle k\left|\left(\rho^{\alpha}\right)^{\prime}\right| k\right\rangle \\
= & \max _{q_{k}, u}\left\{\rho_{11}^{\alpha} \ln q_{1}^{\alpha}+\left(\rho_{22}^{\alpha}\right)^{\prime} \ln q_{2}^{\alpha}\right. \\
& \left.+\left(\rho_{33}^{\alpha}\right)^{\prime} \ln q_{3}^{\alpha}\right\},
\end{aligned}
$$


where $\left(\rho^{\alpha}\right)^{\prime}=u^{\dagger} \rho^{\alpha} u$. This gives

$$
\begin{aligned}
\omega_{\alpha}= & \max _{q_{k}^{\alpha}, \theta_{1}, \theta_{2}}\left\{\rho_{11}^{\alpha} \ln q_{1}^{\alpha}\right. \\
& +\sin ^{2}\left(\theta_{2}\right)\left(\rho_{22}^{\alpha} \ln q_{3}^{\alpha}+\rho_{33}^{\alpha} \ln q_{2}^{\alpha}\right) \\
& +\cos ^{2}\left(\theta_{2}\right)\left(\rho_{22}^{\alpha} \ln q_{2}^{\alpha}+\rho_{33}^{\alpha} \ln q_{3}^{\alpha}\right) \\
& \left.-\sin 2 \theta_{2} \ln \frac{q_{2}^{\alpha}}{q_{3}^{\alpha}} \operatorname{Im}\left[e^{i 2 \theta_{1}} \rho_{23}^{\alpha}\right]\right\}, \\
= & \rho_{11}^{\alpha} \ln \rho_{11}^{\alpha} \\
& +\max _{q_{2}^{\alpha}, \theta_{2}}\left\{\operatorname { s i n } ^ { 2 } \theta _ { 2 } \left(\rho_{22}^{\alpha} \ln \left(1-\rho_{11}^{\alpha}-q_{2}^{\alpha}\right)\right.\right. \\
& \left.+\rho_{33}^{\alpha} \ln q_{2}^{\alpha}\right)+\cos ^{2}\left(\theta_{2}\right)\left(\rho_{22}^{\alpha} \ln q_{2}^{\alpha}\right. \\
& \left.+\rho_{33}^{\alpha} \ln \left(1-\rho_{11}^{\alpha}-q_{2}^{\alpha}\right)\right) \\
& \left.+\sin 2 \theta_{2} \ln \frac{q_{2}^{\alpha}}{1-\rho_{11}^{\alpha}-q_{2}^{\alpha}}\left|\rho_{23}^{\alpha}\right|\right\} .
\end{aligned}
$$

where the optimal values for $q_{1}^{\alpha}=\rho_{11}^{\alpha}$ and $\theta_{1}^{\alpha}=\pi / 2-$ $\phi_{23}^{\alpha} / 2 ; \rho_{23}^{\alpha}=\left|\rho_{23}^{\alpha}\right| e^{i \phi_{23}^{\alpha}}$ are substituted in the above equation. The final expression for the relative entropy of synchronization can be expressed as

$$
\Omega_{\mathrm{R}}=-S(\rho)-\omega_{A}-\omega_{B} .
$$

We plot Eq. (28) in Fig. 4(c). The Arnold tongue is now smaller when compared to the case of coupled spin-1 atoms without local drives in Fig. 4(a), indicating weaker mutual synchronization between the atoms. This is expected as the atoms get entrained to their local drives. In order for the atoms to become mutually synchronized they must now be more strongly coupled to overcome the effect of local entrainment. The inset of Fig. 4(c) shows the difference between $\Omega_{R}(\rho)$ for partially-coherent limit cycle states and mutual information between the spin- 1 atoms.

\section{DISCUSSION AND CONCLUSIONS}

In this manuscript, we introduced a measure of synchronization based on distance to the limit-cycle dynamics. This measure is inspired simultaneously by well known information theoretic measures of entanglement, discord and other quantum correlations and by an understanding of synchronization as the deviation from limit-cycle dynamics. Furthermore, it is often desirable to study synchronization to a single drive in an otherwise complex system, and our measure allows such flexibility by the choice of the limit-cycle dynamics. We capture synchronization and entrainment dynamics of a variety of different systems such as unipartite systems being entrained to external drives, bipartite or multipartite systems that are mutually coupled and driven quantum systems coupled to each other. Our measure allows us to filter the dynamics we are interested in and construct a unified measure of synchronization that applies to finite and infinite dimensional systems identically. We note that since the underlying set of limit-cycle states can be different for different examples, the synchronization measure of two completely disparate systems should not be directly compared. Instead, by making sure that the underlying principles of the measure are the same, we expect that proportional changes in the measure of synchronization are meaningful to discuss.

This measure can be viewed as a generalization of the mutual information theoretic measure introduced in Ref. [13] and hence we comment on critical differences. Firstly, since mutual information is a bipartite measure, it does not accommodate unipartite systems that are entrained to external signals. Likewise, since strong sub-additivity inequality does not hold for generic multipartite systems, the mutual information theoretic measures cannot be applied to multipartite systems. In contrast to this, since the relative entropy is always evaluated between the steady state and the limit-cycle state, our measure is well defined. The relative entropy can be infinite if the support of $\rho_{\text {lim }}$ is different than the support of $\rho$, but this is easily remedied by moving to the trace distance measure of synchronization. Furthermore, mutual information does not allow us to capture complex dynamical systems which involve coupling of locally driven quantum systems. Finally, we note that if we define the limit-cycle states to be the tensor product of the marginal states, we recover the mutual information measure.

Likewise, our measure reduces to the relative entropy of coherence if the underlying set of limit-cycle states is chosen to be the set of diagonal states. This conceptually generalizes the relationship between synchronization and $l_{1}$ norm of coherence presented in [30]. Our measure indeed interpolates between the two scenarios, one where nothing is known about the subsystem dynamics (and hence the marginal states are chosen as the limit-cycle states) and another where a lot is known about the limit-cycle dynamics (that they are diagonal). By interpolating between these two situations, we demonstrate synchronization between dissimilar systems not considered before. In Sec. V B, we investigated mutual synchronization between a three-level atomic degree of freedom and a van der Pol oscillator. Likewise, in Sec. VD, we considered another novel example and studied mutual synchronization between two partially coherent three-level atoms. We hope that our measure will lead to applications of quantum synchronization in hybrid quantum systems such as opto- and nanomechanical systems, vacancy centers coupled to cavities and circuit QED.

\section{ACKNOWLEDGMENTS}

S.V. acknowledges support from an IITB-IRCC Grant No. 16IRCCSG019 and a DST-SERB Early Career Research Award (ECR/2018/000957). M.H. acknowledges support by the Air Force Office of Scientific Research under Award No. FA2386-19-1-4038. 
[1] S. Strogatz, Sync: The Emerging Science of Spontaneous Order (Penguin UK, London, 2004).

[2] A. Pikovsky, M. Rosenblum, and J. Kurths, Synchronization: A Universal Concept in Nonlinear Sciences (Cambridge University Press, New York, 2003), Vol. 12.

[3] A. Balanov, N. Janson, D. Postnov, and O. Sosnovtseva, Synchronization: From Simple to Complex (Springer Science \& Business Media, Heidelberg, 2008).

[4] S. Walter, A. Nunnenkamp, and C. Bruder, Quantum Synchronization of a Driven Self-Sustained Oscillator, Phys. Rev. Lett. 112, 094102 (2014)

[5] T. E. Lee and H. R. Sadeghpour, Quantum Synchronization of Quantum Van Der Pol Oscillators with Trapped Ions, Phys. Rev. Lett. 111, 234101 (2013).

[6] S. Sonar, M. Hajdušek, M. Mukherjee, R. Fazio, V. Vedral, S. Vinjanampathy, and L.-C. Kwek, Squeezing Enhances Quantum Synchronization, Phys. Rev. Lett. 120, 163601 (2018).

[7] T. E. Lee, C.-K. Chan, and S. Wang, Entanglement tongue and quantum synchronization of disordered oscillators, Phys. Rev. E 89, 022913 (2014).

[8] S. Walter, A. Nunnenkamp, and C. Bruder, Quantum synchronization of two van der pol oscillators, Annalen der Physik 527, 131 (2015)

[9] V. M. Bastidas, I. Omelchenko, A. Zakharova, E. Schöll, and T. Brandes, Quantum signatures of chimera states, Phys. Rev. E 92, 062924 (2015).

[10] A. Roulet and C. Bruder, Synchronizing the Smallest Possible System, Phys. Rev. Lett. 121, 053601 (2018).

[11] A. Roulet and C. Bruder, Quantum Synchronization and Entanglement Generation, Phys. Rev. Lett. 121, 063601 (2018).

[12] D. Witthaut, S. Wimberger, R. Burioni, and M. Timme, Classical synchronization indicates persistent entanglement in isolated quantum systems, Nat. Commun. 8, 14829 (2017).

[13] V. Ameri, M. Eghbali-Arani, A. Mari, A. Farace, F. Kheirandish, V. Giovannetti, and R. Fazio, Mutual information as an order parameter for quantum synchronization, Phys. Rev. A 91, 012301 (2015).

[14] A. Mari, A. Farace, N. Didier, V. Giovannetti, and R. Fazio, Measures of Quantum Synchronization In Continuous Variable Systems, Phys. Rev. Lett. 111, 103605 (2013).

[15] T. Weiss, A. Kronwald, and F. Marquardt, Noise-induced transitions in optomechanical synchronization, New J. Phys. 18, 013043 (2016).
[16] G. Manzano, F. Galve, G. L. Giorgi, E. Hernández-García, and R. Zambrini, Synchronization, quantum correlations and entanglement in oscillator networks, Sci. Rep. 3, 1439 (2013).

[17] G. L. Giorgi, A. Cabot, and R. Zambrini, " Transient synchronization in open quantum systems," in Advances in Open Systems and Fundamental Tests of Quantum Mechanics, edited by B. Vacchini, H.-P. Breuer, and A. Bassi (Springer International, Cham, 2019), pp. 73.

[18] A. W. Laskar, P. Adhikary, S. Mondal, P. Katiyar, S Vinjanampathy, and S. Ghosh, Observation of Quantum Phase Synchronization in Spin-1 Atoms, Phys. Rev. Lett. 125, 013601 (2020).

[19] M. Koppenhöfer, C. Bruder, and A. Roulet, Quantum synchronization on the ibm q system, Phys. Rev. Research 2, 023026 (2020).

[20] V. Vedral, M. B. Plenio, M. A. Rippin, and P. L. Knight, Quantifying Entanglement, Phys. Rev. Lett. 78, 2275 (1997).

[21] M. Hajdušek and V. Vedral, Entanglement in pure and thermal cluster states, New J. Phys. 12, 053015 (2010).

[22] M. Hajdušek and M. Murao, Direct evaluation of pure graph state entanglement, New J. Phys. 15, 013039 (2013).

[23] K. Modi, T. Paterek, W. Son, V. Vedral, and M. Williamson, Unified View of Quantum and Classical Correlations, Phys. Rev. Lett. 104, 080501 (2010).

[24] K. Modi, A. Brodutch, H. Cable, T. Paterek, and V. Vedral, The classical-quantum boundary for correlations: Discord and related measures, Rev. Mod. Phys. 84, 1655 (2012).

[25] T. Baumgratz, M. Cramer, and M. B. Plenio, Quantifying Coherence, Phys. Rev. Lett. 113, 140401 (2014).

[26] N. Es'haqi-Sani, G. Manzano, R. Zambrini, and R. Fazio, Synchronization along quantum trajectories, Phys. Rev. Research 2, 023101 (2020).

[27] M. Koppenhöfer and A. Roulet, Optimal synchronization deep in the quantum regime: Resource and fundamental limit, Phys. Rev. A 99, 043804 (2019).

[28] J. R. Johansson, P. D. Nation, and F. Nori, Qutip: An opensource python framework for the dynamics of open quantum systems, Comput. Phys. Commun. 183, 1760 (2012).

[29] J. R. Johansson, P. D. Nation, and F. Nori, Qutip 2: A python framework for the dynamics of open quantum systems, Comput. Phys. Commun. 184, 1234 (2013).

[30] N. Jaseem, M. Hajdušek, V. Vedral, R. Fazio, L.-C. Kwek, and $\mathrm{S}$. Vinjanampathy, Quantum synchronization in nanoscale heat engines, Phys. Rev. E 101, 020201(R) (2020). 